\title{
Mi tío de América, Henri Laborit y la ilustración de la teoría neurobiológica de la organización social
}

\author{
F. Bosch-Lonch, J.E. Baños
}

Los cambios recientes y venideros en la docencia universitaria condicionarán cada vez más el empleo de herramientas nuevas y más originales en la enseñanza. En el ámbito de las ciencias biomédicas en particular existen ya experiencias previas sobre el uso del cine comercial como una manera de favorecer el debate y la formación en áreas y asignaturas diferentes. Este artículo sugiere el empleo de la película Mi tío de América a través de la experiencia en estudiantes de Biología, como una forma de presentar las teorías de Henri Laborit sobre la neurobiología de la organización social. Se trata de una película realizada en 1980, dirigida por Alain Resnais y con guión de Jean Gruault. La trama discurre a lo largo de la vida de tres personajes que viven situaciones diversas que permiten reflejar algunas de las teorías de Laborit. En ellas, básicamente se justifica la conducta humana a través de la jerarquización de tres estructuras cerebrales y niveles, desde el más primitivo y primario -arquiencéfalo-, pasando por el paleoencéfalo, responsable de la memoria, hasta llegar a un nivel más sofisticado responsable de la conciencia, el neocórtex. La película pretende divulgar estas teorías y, para ello, emplea recursos diversos como imágenes de animales de experimentación en situaciones idénticas o similares a las vividas por los protagonistas. Si prestamos atención no sólo a las ventajas que puede ofrecer esta película para su empleo en la docencia sino también a algunas de las limitaciones propias de este filme, se podría concluir que Mi tío de América es altamente recomendable para la enseñanza en psiconeurobiología.

Palabras clave. Aprendizaje. Docencia. Neurobiología. Películas comerciales. Psicobiología.

\section{Mon oncle d'Amérique, Henri Laborit and the illustration of the neurobiological theory of the social organization}

The present and future changes in the teaching at the universities will advise for the use of new and more original methods. In the biomedical sciences there are some experiences regarding the use of popular movies to allow the discussion and interchange of points of view on some polemic topics. This review suggests the usefulness of the film Mon oncle d'Amérique in the teaching of students of human Biology, as a way of introducing the neurobiological theories of Henri Laborit on social organization. This film was directed by Alain Resnais and written by Jean Gruault in 1980. It considers the life of three people who live several situations that are used to explain the Laborit theories. Basically, they justify the human behaviour by the hierarchical organization of the brain in three levels: the most primitive is the archiencephal, the intermediate is the paleoencephal (linked to memory) and the highest, the neocortex that is responsible of consciousness. The movie tries to introduce to these theories using several filmic resources such the viewing of laboratory animals in situations that mimic those lived by the three people. These comparisons allow Laborit himself to explain why people behave in specific ways when submitted to social conflicts. We conclude that Mon oncle d'Amérique is highly recommended for the teaching of some principles related to psychology, psychoneurobiology and psychiatry.

Key words. Learning. Neurobiology. Popular movies. Psychoneurobiology. Teaching.

\section{Introducción}

La utilización de películas comerciales en la docencia universitaria ha adquirido interés en los últimos años [1]. Su empleo tiene ventajas importantes: permite ilustrar situaciones complejas, cuenta con la credibilidad de los recursos cinematográficos, permite contextualizar los problemas y ofrecer diversos puntos de vista [2]. Además, suscita un debate constructivo entre los asistentes que colabora a la consecución de
Departamento de Ciencias Experimentales y de la Salud. Universitat Pompeu Fabra. Barcelona. España.

\section{Correspondencia} Josep-Eladi Baños. Departamento de Ciencias Experimentales y de la Salud. Facultat de Ciències de la Salut i de la Vida. Parc de Recerca Biomèdica de Barcelona. Universitat Pompeu Fabra. Dr. Aiguader, 88. E-08003 Barcelona.

\section{Fax +34935422802 \\ E-mail josepeladi.banos@upf.edu}


los objetivos educativos que llevan a su proyección [3]. En los centros españoles de ciencias de la salud empiezan a existir incluso actividades y cursos que están basados en el empleo de las películas comerciales de forma preferente, tanto en el ámbito de la farmacología [3,4], la medicina [5-7], la farmacia [8] y la biología humana [9]. Con los nuevos aires que aporta el Espacio Europeo de Educación Superior, actividades de este tipo podrían ganar protagonismo en los planes de estudio próximos.

Un problema que se plantea con frecuencia es la elección de la película para ser empleada como método docente. Debe contener un claro mensaje vinculado a los intereses de la titulación, los estudiantes no deben haberla visto previamente (si es posible), debe presentar situaciones que susciten debate y debe favorecer la visión multidisciplinaria de los problemas que se plantean. No siempre es fácil encontrar películas que cumplan estos requisitos, aunque existen fuentes donde tal información está disponible $[10,11]$. Por estas razones, la difusión de experiencias con películas específicas es de notable interés para los docentes interesados en el empleo de este recurso docente. En el presente artículo se revisan las características de Mi tío de América, una película que puede contribuir al debate en torno a la neurobiología de la organización social según las teorías de Henri Laborit.

\section{La película}

Mi tío de América (Mon oncle d'Amérique) [12] es una película dirigida por Alain Resnais y estrenada en 1980. El autor del guión fue Jean Gruault, quien ya había colaborado con importantes directores como François Truffaut (Jules et Jim, L'enfant sauvage), Roberto Rossellini ( $L a$ toma del poder por Luis XIV) y Jean Luc Godard (Les carabiniers). Sus principales intérpretes fueron Gérard Depardieu (René Raguenau), Nicole García (Janine Garnier), Roger Pierre (Jean Le Gall) y Henri Laborit, interpretándose a sí mismo. Laborit, en su momento, también participaría en el guión de la película.

El filme tuvo un notable éxito de crítica y público. Recibió diversos galardones como el del Gran Premio del Jurado a Alain Resnais en el Festival de Cine de Venecia (1980), el del Círcu- lo de Críticos de Cine de Nueva York a la mejor película (1980), el David de Donatello al mejor guión de película extranjera (1981) y el del Sindicato de Críticos de Cine Francés a la mejor película (1981). Fue nominada al Oscar como mejor guión original (1981) y en España fue premiada con el Fotogramas de Plata a la mejor película extranjera.

Técnicamente, la película tiene un interés notable. Se inicia con la explicación de los orígenes familiares de los tres personajes principales y con explicaciones de Henri Laborit sobre el comportamiento. Al principio, la vida de estos tres personajes discurre de forma paralela y semejante desde su independencia familiar, el éxito laboral y una época crítica donde empiezan a entrelazarse los protagonistas. Los conflictos se presentan de forma dramatizada, con frecuentes incursiones de Laborit sobre la conducta animal y escenas en blanco y negro entremezcladas de corta duración de actores del cine francés (Danielle Darrieux, Jean Gabin, Jean Marais). El objeto de este recurso es diverso, pues, en ocasiones, se identifica con los rasgos faciales de los protagonistas; en otras, sirve de introducción inmediata a la secuencia posterior. La historia de Janine y Jean confluyen primero y en la última parte de la película también comparten situaciones.

Las aportaciones científicas de Laborit a través de los experimentos realizados con los animales de laboratorio empiezan a identificarse con las reacciones de los protagonistas, de manera que permiten al investigador establecer una teoría general de conducta de los animales a los seres humanos. Para ilustrarlas aún más, algunas escenas emplean ratas 'humanizadas' que simulan el comportamiento que tendrían los animales frente a las situaciones que plantea la película para los seres humanos.

\section{La figura de Henri Laborit}

La película supuso el conocimiento del público de la figura y de la obra de Henri Laborit (19141995), uno de los padres de la psicofarmacología moderna. Laborit se formó como médico y cirujano militar, y su interés científico debutó con el estudio de la hibernación artificial como método para reducir el shock postoperatorio. En 1949, Laborit era un cirujano militar de la Mari- 
na francesa que ejercía en un hospital tunecino cercano a Bizerta. Había empezado a utilizar los antihistamínicos descubiertos unos años antes para premedicar a los pacientes sometidos a cirugía [13]. La razón que le guiaba en tal decisión era el deseo de prevenir el shock traumático que Laborit atribuía al aumento de la permeabilidad capilar causada por la liberación de histamina. De hecho, Laborit buscaba la mejor combinación para obtener una especie de hibernación artificial que permitiera prevenir el shock quirúrgico [14]. Para ello, añadió mepiramina y prometacina, dos antagonistas $\mathrm{H}_{1}$, a la petidina, que resultó lo que se conoció como cóctel lítico. En los meses siguientes dedujo que los antihistamínicos debían tener una acción central, pues el estado anímico de los pacientes mejoraba, tenían menos ansiedad y precisaban de dosis inferiores de morfina. A pesar de que un psiquiatra militar confirmó las impresiones de Laborit, éste no profundizó en sus observaciones hasta que volvió a la metrópolis poco después.

Tras su traslado a París, Laborit siguió explorando los efectos centrales de los antihistamínicos y descubrió, junto al anestesista Pierre Huguenard, que estos fármacos eran capaces de reducir la temperatura corporal y el metabolismo basal, con lo que podían reducirse las dosis de anestésicos. Convencido de la importancia de sus hallazgos, decidió visitar a los fabricantes de la prometacina, los laboratorios Spezia de Rhône-Poulenc (ahora Aventis) para explicarles su trabajo y pedirles un derivado que tuviera acciones centrales potentes. Fruto de su petición, Paul Charpentier sintetizó la clorpromacina en diciembre de 1950 y la farmacóloga Simone Courvoisier demostró su potencia elevada y toxicidad escasa poco después. Unos meses más tarde, Laborit recibió varias muestras del fármaco que empleó en sus investigaciones clínicas para confirmar que tenía un potente efecto tranquilizante y antishock en pacientes sometidos a cirugía, lo que le sugirió el interés de probarlo en pacientes psicóticos. Así, la clorpromacina se añadió a la petidina y la prometacina en el ya mencionado cóctel lítico, y Hamon demostró su eficacia para reducir la agitación en un paciente psicótico en 1952, pero no le prestó mayor atención, más allá de publicar la observación. La publicación no pasó desapercibida para el psiquiatra francés Pierre Deniker, quien junto a Jean Delay, decidió iniciar el primer ensayo clínico con clorproma- cina en 1955 en pacientes psicóticos con intensa agitación [14]. El fármaco tenía un perfil nuevo: reducía la agitación, la agresividad y las alucinaciones, sin causar somnolencia, y producía una reducción en el movimiento. En 1955, Delay y Deniker acuñaron el término 'neuroléptico' para referirse a los psicofármacos que poseían este efecto [13]. Sin embargo, Delay y Deniker no comentaron la obra previa de Laborit, por lo que es frecuente que se ignore el protagonismo de éste en el descubrimiento de la clorpromacina [15].

En los años siguientes Laborit se entregó al estudio del comportamiento humano a través de la investigación en el laboratorio, especialmente de las conductas agresivas. Escribió numerosas obras para divulgar sus teorías, de las que se han publicado en español El hombre y la ciudad (1973), Del sol al hombre (1973), Introducción a la biología del comportamiento (1975), La paloma asesinada (1986), Dios no juega a los dados (1989) y La vida anterior (1990). Sus contribuciones se reconocieron con importantes distinciones, como el premio Lasker (1957) y el título de oficial de la Legión de Honor (1967).

\section{Las teorías de Laborit en Mi tío de América}

Laborit partía en sus teorías de estudios realizados en animales (etológicos) que después interpretaba de acuerdo a la psicología y el comportamiento de los seres humanos. Sus teorías intentaban relacionar la existencia de estructuras cerebrales jerarquizadas que ayudaban a comprender las diferentes facetas de la conducta humana. Así, defendía la existencia de una organización cerebral en tres niveles: el arquiencéfalo, el paleoencéfalo y el neocórtex. El primero tendría el objetivo principal de asegurar los comportamientos primarios como la ingesta y la reproducción; es el más primario, y Laborit lo asimilaba a los reptiles. El paleoencéfalo, en cambio, sería una estructura mucho más compleja y que se vincularía al recuerdo de aquello que es agradable o desagradable, y asociaba conductas a la pulsión de la recompensa y a la evitación del castigo. Sería aún un nivel inconsciente pero que ayudaría al establecimiento de las sociedades, ya que permitiría su supervivencia al mismo tiempo que obligaría a sacrificar parte de la libertad para mantenerlas. 
El tercer componente, el córtex cerebral, es el más 'humano, e implica la conciencia. Sin embargo, para Laborit la conciencia racionaliza las conductas generadas por el paleocórtex y, de alguna manera, la educación es un proceso que tiende a hacer posible este proceso. En esta teoría global se incluye el inconsciente en un sentido diferente del freudiano, pues considera que no viene desde fuera (sociedad) sino desde dentro (base genética). El aprendizaje supondría una adaptación del individuo a la sociedad, con lo que se beneficia ésta antes que él mismo [16].

Otra de las teorías más interesantes de Laborit que se ilustra en la película es la referida al análisis de las consecuencias que se derivan de la toma de decisiones del individuo frente a una situación específica. Además de la conducta de gratificación asociada a las necesidades asociadas al arquiencéfalo (búsqueda de alimento, procreación), Laborit establece las de gratificación y de inhibición. Las primeras permiten reforzar positivamente aquellas conductas de las que se ha derivado una experiencia placentera. Las segundas, en cambio, generan una conducta de inhibición con las opciones de huida o de agresión. En el ámbito psicopatológico, Laborit establecería en estas situaciones la aparición de ansiedad ante la necesidad de tomar una decisión entre ambas. De hecho, cuanto más abajo en la escala social más posibilidades de que tal dilema aparezca, por lo que los individuos sociales buscan la dominación sobre el otro de forma instintiva como una forma de reducción de tales conflictos. La vida sería pues una búsqueda de estrategias de dominación que se adquirirían a través del aprendizaje (la educación). Para Laborit, tal situación es muy positiva para la sociedad, aunque sea negativa para los individuos que la conforman [16].

Las teorías de Laborit se sustentaban en el análisis de los resultados obtenidos en el laboratorio al exponer a los animales a estímulos aversivos, como ocurre en la jaula de Skinner. Este dispositivo divide el compartimento donde se encuentra el animal en dos partes separadas por una portezuela. Tras una señal acústica, el animal recibe una descarga eléctrica después de una latencia de varios segundos. Tras algunas sesiones, el animal aprende a evitarla atravesando la portezuela a una zona segura donde no la recibirá, con lo cual la situación de distrés se encuentra bajo control y no produce alteración patológica alguna. Cuando la portezuela se cierra, el animal no puede escapar del estímulo aversivo y la consecuencia es la aparición de afecciones por somatización y por inmunodepresión que pueden acabar con la muerte. Si son dos animales los que comparten la situación, esta autoagresión se desvía a una conducta de agresión del otro animal para aliviar la incapacidad de evitar los estímulos displacenteros [16].

Esta represión de la agresividad generada por el proceso de la socialización humana puede interpretarse como un proceso altamente traumático y estresante que es motivo de deterioro biológico y psicológico en los seres humanos que viven en sociedades complejas, como las occidentales [17]. Tal aplicación de una teoría biológica a una interpretación sociológica no deja de tener un interés notable como punto de partida del debate sobre las enfermedades de nuestras sociedades.

\section{La trama de Mi tío de América}

La película se desarrolla en torno a tres personajes con sus propias historias. El primero, Jean Le Gall, es un periodista que ejerce de director de informativos en una importante radio francesa. Proviene de una familia burguesa tradicional e intenta llevar una carrera política y literaria al mismo tiempo. Está casado con su novia de juventud y lleva una vida plácida hasta que descubre a Janine Garnier, una joven actriz, hija de militantes comunistas a los que abandonó para seguir la vida que deseaba. Jean deja a su familia y se va a vivir con Janine, pero su vida se tuerce cuando se le releva de su cargo en la radio sin un motivo aparente. Por su parte, Arlette, mujer de Jean, confiesa a Janine que está gravemente enferma y le pide que abandone a Jean para que éste pueda volver a vivir con ella durante sus últimos meses de vida, y le ruega que no se lo diga a su marido. Janine accede y su vida cambia radicalmente cuando deja el teatro y se convierte en asesora de un grupo empresarial.

El tercer personaje es René. Hijo de una familia católica de agricultores con problemas económicos, decide formarse en el ámbito de la empresa textil y abandonar a sus padres por oponerse a esta elección. La empresa empeora con el tiempo y es obligado a trasladarse a otra lejos de su domicilio. Su esposa, embarazada de su tercer hijo, decide finalmente no acompañarle al nuevo lugar. En uno y otro empleo sufre humillaciones y 
desconsideraciones sobre su trabajo, que llegan al límite cuando se le quiere apartar de nuevo de su trabajo tras un análisis de la baja productividad de la empresa realizado por Janine. René responde mal a la nueva situación, primero adopta una actitud agresiva que más tarde vuelve contra sí mismo con un intento de suicidio. Janine no es más feliz en su vida privada cuando descubre que la mujer de Jean le mintió y que éste, al saberlo, prefiere seguir con ella antes que volver con su amante. Al descubrirlo, Janine responde abofeteando a René.

En realidad, toda la película es una excusa para demostrar el paralelismo entre las teorías de Laborit que él mismo expone ilustrándolas con experimentos de laboratorio. En la segunda parte de la película, los comportamientos de los personajes se identifican con las conductas que son esperables según los experimentos animales. Éstos se emplean para ilustrar las razones que pueden explicar por qué los personajes sufren en su vida personal por las circunstancias a las que deben enfrentarse.

La película acaba con unas palabras de Laborit sobre el inconsciente, definido como aquello que el hombre desconoce (prejuicios, juicios de valor), pero que condiciona su comportamiento y el establecimiento de la escalas jerárquicas de dominación. En su opinión, sin el conocimiento de los mecanismos cerebrales ningún cambio será posible. Una obra reciente [18] ha destacado la reflexión de vigencia actual que Laborit introdujo en el filme: 'Para poder llegar a la luna, la humanidad tuvo que conocer primero la fuerza de la gravedad; eso no significa que ésta haya dejado de existir sino que puede dominarse. Del mismo modo, mientras la humanidad no difunda tanto como sea posible entre todos los habitantes del planeta, la manera cómo funciona nuestro cerebro, el modo cómo lo utilizamos, y hasta que no sepamos que siempre se ha empleado para dominar a los demás, hay pocas posibilidades de que las cosas cambien'.

Finalmente, el porqué del título. Los tres personajes en un momento u otro de la película recuerdan al 'tío de América' como aquel que ha de ayudarles a solucionar sus problemas, el símbolo de algo fuera de ellos que puede solucionar sus situaciones sin que deban tomar las decisiones por sí mismos, y muy probablemente como el sueño de lo que hubieran querido ser, es decir, como el símbolo del éxito. La vida real no puede continuar sin eliminar esta ficción y se trata de conocer las reglas de conducta para saber cómo poder vivir en una sociedad donde no es posible eliminarlas [19].

\section{Interés de la película como instrumento docente}

En primer lugar, debe decirse que Mi tío de América no es una película sencilla y que puede precisar de más de una visión para encontrar el entramado que esconde. Ello no es óbice para recomendarla a los estudiantes de ciencias de la salud en general y para los de medicina, psicología y sociología en particular. Laborit ilustra perfectamente diversos aspectos de la neurobiología que relaciona con el comportamiento humano y las complejidades de las relaciones sociales.

En el ámbito neurobiológico cabe destacar la teoría sobre los diferentes comportamientos, como el de consumo, el de gratificación o el de inhibición, con las respuestas de lucha o huida. En este sentido es importante la vinculación de la ausencia de respuesta adaptativa ('controladora' de la situación) con la aparición de ansiedad, angustia en palabras de Laborit. Esa ansiedad conlleva la aparición de conductas de 'autoagresión' en las situaciones en las cuales el individuo no puede enfrentarse adecuadamente a ellas. Resnais utiliza el empeoramiento de la úlcera gastroduodenal de René Ragueneau para explicarla de forma explícita. Menos evidente es la aparición de un cólico nefrítico en Le Gall tras ser despedido de su empleo en la radio. Todos estos conflictos son ilustrados por Laborit mediante la presentación de los clásicos experimentos en una jaula de Skinner, donde se muestra el empeoramiento de la salud de los animales sometidos a estímulos eléctricos aversivos de los que no pueden escapar.

Laborit presenta también su opinión sobre los aspectos sociológicos que pueden explicarse por razones psicológicas. Para él, la vida en sociedad es esencial porque asegura la supervivencia del individuo y, obviamente, en último término, de la propia sociedad. Las razones para explicarlo provienen de la transmisión del conocimiento a través del lenguaje y de que la supervivencia del individuo está ligada al aprendizaje de lo que es adecuado y de lo que no para mantener la cohesión del grupo. Así, se castiga o premia aquello que es eficaz para la supervivencia de la sociedad y el lenguaje oculta las situaciones de jerarquía 
que subyacen en tal adecuación. En definitiva, se prioriza la sociedad al individuo con las consecuencias que tiene tal decisión para el último.

Un ejemplo de esta teoría es la explicación que Laborit presenta mediante el empleo de nuevo de la jaula de Skinner. Cuando los animales son sometidos a estímulos aversivos inevitables, su salud se resiente por un aumento de su vulnerabilidad a las enfermedades. Ello se debe a un exceso de actividad vegetativa y a una inmunodepresión derivadas ambas de la ansiedad generada por la falta de control sobre la situación. Los animales pueden generar conductas agresivas que en seres humanos se consideran desadaptadas, por lo que acaban reprimiéndose. La consecuencia es el desvío de esa heteroagresión a una autoagresión que puede tener consecuencias funestas. Así, en un principio pueden aparecer alteraciones psicosomáticas (úlcera gastrointestinal, hipertensión, infarto de miocardio) que pueden llevar incluso al suicidio, la expresión máxima de esa autoagresión.

La sesión cinematográfica también puede servir para incidir en la introducción de los fármacos antihistamínicos y antipsicóticos. Como se ha comentado en el resumen biográfico de Laborit, éste contribuiría no sólo al empleo de la prometacina para potenciar la anestesia, sino también a la introducción clínica de la clorpromacina [20], que más tarde conllevaría a su empleo en psiquiatría.

Nuestra experiencia con Mi tío de América en docencia supone la continuación de una tradición en el empleo de películas comerciales para complementar la formación de estudiantes de disciplinas diferentes, desde asignaturas de créditos de libre elección organizadas desde la Facultad de Medicina de la Universitat Autònoma de Barcelona [3], hasta asignaturas de quinto curso de Biología en la Universitat Pompeu Fabra [9]. En esta ocasión se proyectó el filme entre alumnos de la licenciatura de Biología, a los que previamente y por grupos se les pidió que prestaran especial atención a determinados aspectos de la película: el perfil de los tres protagonistas, el del propio Laborit, sus teorías y los recursos cinematográficos que continuamente emplea Resnais.

Cabe señalar que la experiencia fue positiva en términos generales, aunque es preciso des- tacar la existencia de algunos puntos en contra que convendría tener en cuenta por parte de los docentes que se decidiesen por el empleo de $M i$ tío de América entre sus estudiantes. A la ya citada complejidad del filme, cabría añadir su larga duración. Probablemente para proyecciones de unas dos horas sea recomendable programar una pausa corta para el descanso del auditorio. La antigüedad de la película suele ir injustamente en contra de muchos estudiantes que acostumbran a ver con mala cara todo lo que consideran anticuado. Por otro lado, el reflejo evidente de una sociedad francesa tradicional de la época con tendencias también puede herir ciertas sensibilidades feministas entre el alumnado. Por último, una formación científica consolidada por parte de los estudiantes puede contrastar con el abordaje, a veces algo superficial y simplista, de las teorías del comportamiento humano tal como las refleja Resnais en la película.

A pesar de todo, la mayoría de los estudiantes valoraron como positiva la proyección de la película según se recogió en los informes que redactaron después de la actividad. Por todo ello, los autores consideran que Mi tío de América es un filme rico y complejo, recomendable para hablar de temas inusualmente importantes y complicados. En quizás el único artículo en el que se sugiere el interés de esta película como instrumento docente, Corliss escribía poco después de su estreno: 'Imbedded in the memory of every living thing, and reinforced from the earliest years by parental instruction and example, are the codes that determine how that creature will act. At birth, as gift from the most primitive part of his brain, a child knows the elements of survival: he must eat, drink and reproduce. His early life is filled with the imposition of rituals: toilet training, religious instruction, social communication and compromise. By the time he is an adult, he knows most of the games people play: how to dress and cook, shake hands, argue with a colleague, plead with a lover, break things, break up, make up, attack, escape or withdraw. In each "free action", he is replaying the history of the race as stage-managed by an eons-old brain that wants simply to survive and conquer' [19]. 


\section{Bibliografía}

1. García-Sánchez JE, Trujillano-Martín I, García-Sánchez E. Medicina y cine. ¿Por qué? J Med Movies 2005; 1: 1-2.

2. Baños JE, Bosch F. Las películas comerciales en la docencia de la medicina: más cine, por favor. Rev And Pat Digest 2005; 28: 10-3.

3. Baños JE, Bosch F. Medicamentos, ciencia y sociedad o cómo enseñar farmacología a estudiantes universitarios de cualquier licenciatura. Educ Med 1999; 2: 202.

4. Farré M, Bosch F, Roset PN, Baños JE. Putting clinical pharmacology in context: the use of popular movies. J Clin Pharmacol 2004; 44: 30-6.

5. García-Sánchez JE, Fresnadillo MJ, García-Sánchez E. El cine en la docencia de las enfermedades infecciosas y la microbiología clínica. Enferm Infecc Microbiol Clin 2002; 20: 403-6.

6. Loscos J, Baños J, Loscos F, De la Cámara J. Medicina, literatura y medicina: una experiencia en la Universitat Autònoma de Barcelona. J Med Movies 2006; 2: 138-42.

7. Medicina y Cine. Universidad de Oviedo. URL: http:// www.uniovi.es/Vicerrectorados/Extension/cursos/ extension/20052006/cuatrimestre1/sovied04.htm. [15.12.2006].

8. Escubedo E. Fàrmaco-cinema: aprenentatge de la farmacologia mitjançant el cinema. Conferencia presentada dentro de la sesión de Docencia en Farmacología, en la sede de la Societat Catalana de Farmacologia (ACMCB). Barcelona, España, 28 de junio de 2006.
9. Baños JE, Aramburu J, Sentí M. Biocinema: la experiencia de emplear películas comerciales con estudiantes de Biología. J Med Movies 2005; 1: 1: 42-6.

10. New York State University. Literature, Arts and Medicine Database. URL: http://endeavor.med.nyu.edu/lit-med/litmed-db/about.html. [12.12.2006].

11. García-Sánchez JE, García-Sánchez E. Dos años de Revista de Medicina y Cine. Balance y punto seguido. Rev Med Cine 2006; 2: 123-4.

12. Mi tío de América. Andrea Films TF1. Editada por Vella Vision S.L.; 2005.

13. Sneader W. Drug discovery. A history. Chichester: John Wiley; 2005.

14. Chast F. Histoire contemporaine des médicaments. Paris: Éditions La Découverte; 1995.

15. López-Muñoz F, Álamo C, Cuenca E. Fármacos antipsicóticos. En López-Muñoz F, Álamo C, eds. Historia de la neuropsicofarmacología. Madrid: Eurobook; 1998. p. 207-43.

16. URL: http://es.wikipedia.org/wiki/Henri_Laborit. [01.12.2006].

17. URL: http://ilhn.com/ediciones/2949.html. [01.12.2006].

18. Figueras A. Optimizar la vida. Claves para reconocer la felicidad. Barcelona: Alienta Editorial; 2006.

19. Corliss R. The brain game. Time, 8 de diciembre de 1980.

20. Laborit H, Huguenard P, Allaume R. Un nouveau stabilisateur végétatif (LE 4560 RP). Presse Méd 1952; 60: 206-8. 